\title{
"BIENVENUE EN FRANCE"... AUX RICHES ÉTUDIANTS ÉTRANGERS
}

\author{
Hugo Bréant et Hicham Jamid
}

GISTI | « Plein droit »

2019/4 n 123 | pages 11 à 14

ISSN 0987-3260

Article disponible en ligne à l'adresse :

https://www.cairn.info/revue-plein-droit-2019-4-page-11.htm

Distribution électronique Cairn.info pour GISTI.

(c) GISTI. Tous droits réservés pour tous pays.

La reproduction ou représentation de cet article, notamment par photocopie, n'est autorisée que dans les limites des conditions générales d'utilisation du site ou, le cas échéant, des conditions générales de la licence souscrite par votre établissement. Toute autre reproduction ou représentation, en tout ou partie, sous quelque forme et de quelque manière que ce soit, est interdite sauf accord préalable et écrit de l'éditeur, en dehors des cas prévus par la législation en vigueur en France. Il est précisé que son stockage dans une base de données est également interdit. 
« Bienvenue en France » : le gouvernement a choisi de nommer ainsi sa dernière « stratégie d'attractivité pour les étudiants étrangers ». À cette occasion, les droits d'inscription à l'université pour les nonressortissant-es de l'Union européenne ont été fortement augmentés. Cette mesure s'inscrit dans la droite ligne de politiques migratoires et consulaires restrictives. L'intention de réduire le nombre d'étudiant-es venant d'Afrique, implicite depuis quelques années, prend forme avec une véritable politique de sélection par l'argent.

\section{"Bienvenue en France"... aux riches étudiants étrangers}

Hugo Bréant, post-doctorant (ANR Global Youth, IHMC-ENS) et docteur en science politique (Paris 1, CESSP), Hicham Jamid, doctorant-Ater (LISECNRS/Cnam-Paris Q ORMES, université. Ibn Zohr d'Agadir)

Pour l'année universitaire 2017-2018, plus de 343000 étudiantes et étudiants étrangers sont inscrits dans les différents établissements d'enseignement supérieur français ${ }^{1}$. Le Premier ministre vise les 500000 étudiant·es à l'horizon 2027. Il a donc lancé, en novembre 2018, une nouvelle stratégie appelée « Bienvenue en
France - Choose France $»^{2}$, en fixant trois objectifs. Le premier consiste à offrir de meilleures conditions d'accueil en s'engageant à simplifier l'octroi des visas pour études en France, à faciliter l'accès aux logements et à tripler le nombre de bourses attribuées. Le deuxième tient dans le renforcement du rayonnement de l'enseignement français à l'étranger. Le gouvernement veut développer davantage son offre de formation, en délocalisant des antennes d'universités et d'écoles hors de France ${ }^{3}$.

Le dernier objectif est celui qui a suscité le plus de réactions : l'augmentation substantielle des droits d'inscription des seul·es étudiant·es non communautaires. Ainsi, à compter de la rentrée universitaire 2019-2020, ils et elles doivent s'acquitter de 2770 euros pour s'inscrire en licence (contre 170 euros auparavant) et de 3770 euros pour un master (au lieu de 243 euros); soit des frais d'inscription 16 fois plus élevés que pour leurs homologues européens. Dans le rapport remis en février 2019 à la ministre de l'enseignement supérieur, une mission de concertation recommandait de ne pas étendre la hausse aux doctorant·es, dans la mesure où la France apparaît « en voie de décro- 
chage par rapport aux grands pays de recherche ${ }^{4} \gg$.

À bien des égards, la stratégie « Bienvenue en France » peut apparaittre paradoxale, en cherchant à attirer des étudiantes et étudiants étrangers tout en leur faisant payer plus cher leurs études. Et ce d'autant plus quand l'on sait que les promoteurs de la réforme identifient eux-mêmes «la complexité des procédures administratives » et le «coût de la vie élevé, notamment le coût du logement » comme des «freins》 à «l'attractivité internationale » de la France 5 . Mais cet apparent paradoxe prend tout son sens quand on le réinscrit dans le temps plus long des politiques migratoires françaises. Les exigences méritocratiques qui promeuvent «des critères d'excellence académiques », les préoccupations utilitaristes qui guident une politique d'immigration « choisie » et les logiques sécuritaires qui régissent le contrôle des frontières ne fonctionnent pas les unes sans les autres. Les étudiantes et étudiants étrangers demeurent des étrangers avant tout, et leur sélection s'effectue à l'aune de ces trois logiques imbriquées. L'instauration de «Bienvenue en France » engendre une sélection par l'argent qui permet d'attirer les riches, tout en excluant les pauvres.

\section{Attirer les un-es, discriminer les autres}

Alors que, jusqu'aux années 1970, la certification d'un diplôme autorisait les jeunes étrangers à venir étudier en France, le gouvernement mettait fin à cette situation dès 1979 en instaurant une série de conditions : pré-inscription universitaire, test de langue, conditions de ressources, engagement à repartir au terme des études ${ }^{6}$. Les contrôles étaient renforcés pour s'assurer que ces jeunes ne chercheraient pas à travailler pendant ou après leurs études ${ }^{7}$. Dès 2007, consigne était donnée de favoriser les entrées qui «répondent aux besoins économiques » du pays ${ }^{8}$. En 2011, la circulaire Guéant augmentait le seuil de ressources nécessaires au départ, en cherchant à diminuer la délivrance des visas étudiants et en demandant aux préfets de complexifier encore le passage du statut d'étudiant à celui de travailleur'.

La stratégie d'attractivité sélective prônée par «Bienvenue en France » «va encore plus loin dans la sélection ${ }^{\mathbf{1 0}} \gg$, en faisant se rejoindre deux logiques discriminatoires distinctes : l'origine sociale et l'origine géographique. À cet égard, la situation des étudiant·es africain'es est révélatrice ${ }^{11}$. Dans le monde, un·e étudiant.e mobile sur dix est originaire du continent africain ${ }^{12}$. En France, le taux s'établit à presque un sur deux. Sur les 434000 Africain·es qui étudiaient à l'étranger en 2014, la France en accueillait près d'un tiers : 76500 originaires du Maghreb et 65600 d'Afrique subsaharienne. La délivrance des visas pour études a connu une augmentation constante: en 1998, 30000 étaient délivrés, plus de 85000 en 2015. Pourtant, en 2006, le taux de refus de visa étudiant s'élevait à $20 \%$, contre $10 \%$ pour l'ensemble des demandes ${ }^{13}$. Dès 2011, l'Afrique a été la première région d'origine des étudiant·es confrontée à une diminution des visas pour études et, donc, des effectifs étudiants ${ }^{14}$. Si le nombre d'étudiant.es africain.es partant étudier à l'étranger ne cesse d'augmenter, la part de celles et ceux accueillis en France ne cesse de décroître, passant d'un tiers il y a une quinzaine d'années à moins d'un quart aujourd'hui. D'après Campus France, en 2017, 77 \% des étudiant·es africain'es en France s'inscrivent à l'université, très majoritairement en licence et en master, et sont donc très largement concernés par cette réforme. Pourtant, alors que six pays africains font partie des dix pays qui envoient le plus d'étudiant·es en France, Édouard Philippe ne mentionne presque jamais l'Afrique dans son discours, et déclare que les «étudiants indiens, russes, chinois seront plus nombreux et devront l'être ». Quant aux étudiant·es africain·es, ils et elles se verront proposer des bourses d'études ou des exonérations par le ministère des affaires étrangères - renforçant ainsi l'image d'immigré es pauvres, qui méritent assistance -, et seront encouragé es à s'inscrire dans les campus franco-africains que le gouvernement français veut multiplier dans leur pays d'origine.

\section{Étudier en France crève la poche}

Édouard Philippe justifie cette politique d'attractivité par ces mots: "Un étudiant étranger très fortuné qui vient en France paye le même montant qu'un étudiant français peu fortuné dont les parents résident, travaillent et payent des impôts en France depuis des années. C'est absurde et injuste. » Le

Premier ministre oublie que venir étudier en France ne se résume pas à payer des frais d'inscription mais s'inscrit dans un processus long, qui commence aux portes des consulats français ${ }^{15}$. Avant 2010, l'examen des dossiers était effectué par trois acteurs: les agents consulaires chargés de limiter les départs, les membres des services culturels des ambassades désireux de renforcer l'influence culturelle française et les universités françaises espérant garder le contrôle des inscriptions.

Dès 2010, ces critères de sélection ont été codifiés par l'agence Campus France. Établissant le lien entre les universités et les consulats, ses antennes guident les étudiant·es 
dans leurs démarches de pré-inscription. L'agence contrôle ensuite le dossier, émet un avis consultatif et le dépose au consulat, en lieu et place de l'étudiant·e. Cet avis se fonde sur trois critères: la qualité du parcours scolaire, la cohérence du projet d'études et la tangibilité des perspectives professionnelles visées au retour. Campus France participe de fait à la démultiplication « des filtres et des mécanismes de sélection » en amont du consulat ${ }^{16}$.

Les frais de dossier (99 euros pour un visa long séjour) ne constituent que la partie émergée des coûts multiples qui s'additionnent au cours de la procédure. Pour solliciter Campus France, il faut fournir: un acte de naissance, un certificat de nationalité, une carte d'identité et un passeport. L'obtention de ces pièces nécessite le paiement d'une dizaine d'euros, l'accès à une connexion internet, la réalisation de photographies d'identité conformes, la duplication des pièces justificatives, etc., ce qui peut s'avérer particulièrement coûteux surtout quand on habite loin de la capitale ou d'une grande ville, et qu'il faut multiplier les $\gg$ Pour obtenir son visa pour études, il faut posséder d'importantes ressources, tant matérielles qu'immatérielles Isociales, scolaires, linguistiques et symboliques). l'hébergement sont strictes: il doit justifier d'une situation financière stable et de conditions d'hébergement convenables. Bien souvent, les agents consulaires se montrent suspicieux face à l'offre d'hébergement d'un proche immigré, et de plus en plus d'étudiant-es cherchent alors, avant même le départ, à louer un appartement à leur nom. Mais il faut surtout pouvoir justifier de «moyens d'existence » suffisants. Les agents consulaires exigent a minima un apport personnel de 615 euros mensuels ou le soutien d'un proche, qui doit justifier de son lien de parenté et présenter des relevés bancaires indiquant que la somme totale (7380 euros) est bloquée sur un compte ou que des virements mensuels sont planifiés. Autrement dit, pour pouvoir venir étudier en France, il faudra débourser (hors inscription universitaire) près de 10000 euros pour un visa de 12 mois, un coût souvent rédhibitoire dans les pays subsahariens où le salaire moyen n'excède guère quelques dizaines d'euros, et où le taux de bancarisation atteint à peine $20 \%$ de la population. Si la situation économique est relativement plus avantageuse au Maghreb, elle allers-retours en transports collectifs. ne place pas les demandeurs en Par la suite, il faut aussi souscrire une assurance voyage rapatriement; fournir les justificatifs d'une réservation d'un vol aller-retour; attester d'un hébergement pendant la durée du séjour: réservation hôtelière de trois mois maximum ou attestation d'accueil par un particulier. L'appui dans la recherche de logement est fondamental. Mais là encore, les conditions imposées au garant de position beaucoup plus favorable. Bien souvent, les étudiant·es et leurs familles doivent donc épargner sur le temps long, organiser des cotisations dans un cercle familial élargi, voire sacrifier une partie de leur patrimoine (im)mobilier.

Pour espérer obtenir son visa pour études, il faut donc posséder d'importantes ressources, tant matérielles qu'immatérielles (sociales, scolaires, linguistiques et symboliques) ${ }^{17}$. L'augmentation des frais d'inscription universitaire et, plus généralement, des coûts de la mobilité étudiante prouve que, paradoxalement, l'« hospitalité » bien ordonnée commence par les plus riches.

\section{«Bienvenue en France 》 ou l'effet boomerang}

Prenant l'exemple du Danemark, de la Suède ou encore du Royaume-Uni qui ont appliqué des frais de scolarité différenciés pour les étudiant-es non communautaires, la Cour des comptes a publié le 23 novembre 2018 un rapport dans lequel elle pointe la baisse considérable de leurs flux d'arrivée dans ces pays et l'«éventuel effet d'éviction 》 qui peut «dissuader certains étudiants de venir étudier en France ».

Avec « Bienvenue en France », cet «effet d'éviction » devient réalité. Après la clôture de la procédure de demande d'admission préalable (DAP) en première année de licence dans les universités publiques françaises pour la rentrée 2019-2020, Campus France a édité le 5 février 2019 un premier point d'étape qui révèle « une baisse de $10 \%$ des candidats ayant soumis leur dossier ». Deux semaines plus tard, un second communiqué constate cette fois une «quasistabilité » du nombre de dossiers reçus en 2019, avec une chute d'à peine $2,4 \%$ par rapport à 2018 , un chiffre sujet à caution ${ }^{18}$.

Au sein de l'université Paris 8 Vincennes - Saint-Denis, l'effet de cette réforme est particulièrement éloquent. La seule étude menée au niveau d'une université française y a été réalisée avant la rentrée 2019. $2020^{19}$. Si nous ne disposons pas du nombre exact des étudiant·es inscrit·es et réellement arrivées à Paris 8 après cette rentrée, la baisse 
des demandes d'admission des étudiant·es non communautaires transmises par Campus France en avril 2018 et 2019 est «dramatique », de l'ordre de moins $48 \%$. D'après cette enquête, « cette baisse touche de manière plus significative les inscriptions en master (- $57 \%$ ) qu'en licence $(-45 \%)$ ».

Toutes les mesures de hausse des droits d'inscription pour les étudiantes et étudiants étrangers dans des pays européens ont eu des conséquences négatives. Le Conseil constitutionnel - saisi par des associations étudiantes - a affirmé que «l'exigence constitutionnelle de gratuité s'applique à l'enseignement supérieur public » et que les frais d'inscription doivent rester «modiques ${ }^{20}$. Si certain.es se sont réjoui·es de cette annonce, y voyant une « victoire » de leur mobilisation, d'autres sont plus sceptiques quant à l'interprétation du terme «modiques »... sur lequel devra se prononcer le Conseil d'État!

Nos recherches sur le coût des études en France et sur les pratiques mobilisées pour les contourner ${ }^{21}$ montrent que toutes les mobilités étudiantes en France ont pour corollaires la vulnérabilité financière, l'épreuve de discriminations racistes et sexistes, mais aussi la précarité juridique inhérente à leur statut. Souvent originaires de milieux modestes, nombreux sont les étudiants et étudiantes à dépendre des ressources financières de leur famille pendant toute la durée de leur expatriation en France.

Si les différents rapports et travaux de recherche mobilisés dans ce texte convergent pour mettre en garde le gouvernement français contre une application des frais de scolarité différenciés pour les étudiant·es non communautaires, la concrétisation de la stratégie «Bienvenue en France » a levé le voile sur l'ambiguïté de la politique française à l'égard des étudiantes et étudiants étrangers, oscillant entre une stratégie d'attractivité et une logique sécuritaire de mâ̂trise des flux migratoires, voire de contrôle de leurs origines, aussi bien géographiques que sociales. De plus, si les chiffres montrent que ces étudiant es rapportent plus qu'elles et ils ne coûtent à l'État français ${ }^{22}$, l'adoption de cette stratégie dévoile, aussi, le cynisme politique presque assumé de ce gouvernement.

1 Campus France, « Chiffres clés », mars 2019.

2 Édouard Philippe, «Présentation de la stratégie d'attractivité pour les étudiants internationaux », 19 novembre 2018.

3 Pascal Brindeau et Marion Lenne, Rapport d'information sur l'accueil des étudiants étrangers en France, $n^{\circ} 1763$, Assemblée nationale, 13 mars 2019, p. 17.

4 Julien Blanchet et al., Mission de concertation sur la stratégie « Bienvenue en France », Rapport remis à la ministre de l'Enseignement supérieur, de la Recherche et de l'Innovation, février 2019.

${ }^{5}$ Dossier de presse, précité note 2.

${ }^{6}$ Serge Slama, La fin de l'étudiant étranger, L'Harmattan, 1999.

7 Victor Borgogno et Jocelyne Streiff-Fénart, «L'accueil des étudiants étrangers en France: politiques et enjeux actuels », Cahiers de l'Urmis, $\mathrm{n}^{\circ} 5,1999$, p. $77-86$

${ }^{8}$ Anne-Catherine Wagner, «Attirer les talents internationaux. Les ambiguittés d'une hospitalité sélective », Savoir/Agir, 2, 36, 2016, p. 33-38.

${ }^{9}$ Même si la circulaire a été abrogée en 2012, il reste nécessaire d'obtenir une promesse d'embauche et le paiement par l'employeur de la taxe liée au recrutement. Gisti, «Étudiant.e.s., tri sélectif », Plein droit, ${ }^{\circ} 91,2011$, p. 1-2.

10 Lama Kabbanji, « Le plan Bienvenue en France: nouveau volet d'une politique migratoire sélective », De Facto, $n^{\circ}$, mars 2019.

${ }^{11}$ Françoise Blum, «Étudiants africains en France: une si longue histoire... sans avenir? », De Facto, $\mathrm{n}^{\circ} 5$, mars 2019

${ }^{12}$ Campus France, « La mobilité internationale des étudiants africains », Les Notes, Hors-série, $n^{\circ} 16$, novembre 2017 .

${ }^{13}$ Réseau européen des migrations, ministère de l'intérieur, L'immigration des étudiants étrangers en France, 2012; Sarah Belaïsch et Laura Petersell (dir.), Visa refusé. Enquête sur les pratiques des consulats de France en matière de déliurance des visas. Rapport d'observation, La Cimade, 2010.

14 EduFrance, « Les étudiants étrangers en France: évolution et comparaison avec d'autres pays », Lettre d'EduFrance, 2006.

15 Hicham Jamid, « "Bienvenue en France" aux étudiants étrangers, vraiment? », The Conversation, 20 novembre 2018.

${ }^{16}$ Alexis Spire, « Contrôler et choisir. La sélection des étudiants candidats à l'émigration vers la France », in Sylvie Mazzella (dir.), La mondialisation étudiante. Le Maghreb entre Nord et Sud, Karthala, 2009, p. 79-96.

${ }^{17}$ Hugo Bréant, « Étudiants africains: des émigrés comme les autres. Sélectivité sociale du visa et (im) mobilités spatiales des étudiants internationaux comoriens et togolais $\gg$, Politix, $\mathrm{n}^{\circ} 123$, 2018, p. 195-218.

18 Thomas Stoll et Gérald Tenenbaum, « Le Ministère de l'Enseignement Supérieur a manipulé les chiffres de Campus France », Blog Mediapart, 7 avril 2019.

19 Beng Gaëlle et al., « Malvenues en France », recherche collective dirigée par Hélène Nicolas, réalisée dans le cadre du cours « Enquête collective de terrain », Master 1 et 2 en Étude du genre, 2019.

${ }^{20}$ Conseil constitutionnel, décision n²019-809, QPC du 11 octobre 2019.

${ }^{21}$ Hicham Jamid, «Étudier et travailler en France: un développement humain au risque de l'irrégularité du séjour », Migrations Société, 30, 171, 2018, p. 63-78.; Hugo Bréant, op. cit.

${ }^{22}$ Campus France, «Au-delà de l'influence: l'apport économique des étudiants étrangers en France », Les Notes, novembre 2014. 